\title{
Politique
}

Politique

\section{L'engagement intellectuel : mélanges en l'honneur de Léon Dion sous la direction de Raymond Hudon et Réjean Pelletier, Sainte-Foy, Les Presses de l’Université Laval, 1991, 593 p.}

\section{André Bernard}

Numéro 22, automne 1992

URI : https://id.erudit.org/iderudit/040732ar

DOI : https://doi.org/10.7202/040732ar

Aller au sommaire du numéro

Éditeur(s)

Société québécoise de science politique

ISSN

0711-608X (imprimé)

1918-6584 (numérique)

Découvrir la revue

Citer ce compte rendu

Bernard, A. (1992). Compte rendu de [L'engagement intellectuel : mélanges en l'honneur de Léon Dion sous la direction de Raymond Hudon et Réjean Pelletier, Sainte-Foy, Les Presses de l’Université Laval, 1991, 593 p.] Politique, (22),

141-142. https://doi.org/10.7202/040732ar d'utilisation que vous pouvez consulter en ligne.

https://apropos.erudit.org/fr/usagers/politique-dutilisation/ 


\section{RECENSIONS}

L'engagement intellectuel : mélanges en I'honneur de Léon Dion sous la direction de Raymond Hudon et Réjean Pelletier, Sainte-Foy, Les Presses de l'université Laval, 1991, 593 pages.

Ouvrage superbe, L'engagement intellectuel : mélanges en I'honneur de Léon Dion est un éloquent témoignage d'amitié de personnes qui ont été disciples ou camarades de l'un des universitaires les plus remarquables du Québec contemporain. L'exceptionnelle qualité de la plupart des textes réunis dans ce recueil impeccable illustre admirablement la contribution de Léon Dion à la vie intellectuelle au Québec.

Quelques textes, une dizaine, expriment les sentiments de personnes qui ont tenu à dire ce qu'elles avaient le plus aimé chez Léon Dion. Ces textes, qui couvrent une soixantaine de pages, ont été rédigés par Michel Gervais (préface), John Meisel («Léon Dion : I'homme et sa société"), Jean-Guy Paquet, Georges Lavau, Yvon Daneau, Jean-Paul L'Allier, Claude Ryan, Michel Roy et Gilles Lesage, Solange Chaput-Rolland, Antoine Ambroise et, enfin, Claire L'Heureux-Dubé. Ces textes évoquent la personnalité attachante de Léon Dion : ils décrivent I'homme.

Les autres textes se rapportent à son œuvre. Ils présentent des réflexions, qui prolongent ses écrits. La plupart de ces contributions devraient d'ailleurs faire partie des lectures obligatoires dans plusieurs cours professés en science politique. Ce sera assurément le cas des chapitres consacrés à l'analyse systémique (textes de David Easton, de Vincent Lemieux, d'André$J$. Bélanger, de Jean A. Laponce et, enfin, de Jacques-Yvan Morin). Ce sera aussi le cas de plusieurs des chapitres regroupés dans la deuxième partie (Culture et société), écrits par Yvan Simonis, Fernand Dumont, Guy Rocher, Charles Taylor, Micheline de Sève, Pierre Noreau et, enfin, Hugh G. Thorburn. Ce sera même le cas de quelques-uns des chapitres consacrés à divers aspects du nationalisme (troisième partie) ou aux facettes diverses de l'engagement intellectuel (quatrième partie), chapitres rédigés par Louis Balthazar, Stéphane Dion (fils de Léon Dion), Guy Laforest, Michael Oliver, H. Blair Neatby, André Blais et Jean Crête (ces deux anciens disciples de Léon Dion expliquant pourquoi l'opinion 
publique au Canada anglais a rejeté I'Accord du lac Meech), Mildred A. Schwartz, Gérard Pelletier, Gérard Bergeron et, enfin, Bernard Fournier. L'ensemble de ces vingt-deux chapitres (p. 41491) montre la diversité des travaux de Léon Dion et chacun d'eux constitue, dans le prolongement de ces travaux, un apport nouveau aux connaissances.

La lecture de ces textes, exceptionnellement enrichissante, est facilitée par l'excellente présentation qu'en font Raymond Hudon et Réjean Pelletier (p. 1-38), les deux collègues de Léon Dion qui ont coordonné la publication de l'ouvrage.

L'ouvrage présente, à la toute fin (p. 569-585), la liste des publications de Léon Dion. Cette liste est impressionnante : un demi-douzaine de livres, une cinquantaine de contributions à des recueils, et près de deux cents articles de revues, articles de journaux, préfaces et communications diverses. La variété des sujets auxquels s'est intéressé Léon Dion explique le titre de l'ouvrage qui lui est consacré : L'engagement intellectuel. Cet universitaire exceptionnel méritait I'hommage exceptionnel que constitue L'engagement intellectuel : mélanges en I'honneur de Léon Dion. 